\title{
Microlensing of an Elliptical Source by a Point Mass
}

\section{Citation}

Heyrovsky, David, and Abraham Loeb. 1997. "Microlensing of an Elliptical Source by a Point Mass." The Astrophysical Journal 490 (1): 38-50. https://doi.org/10.1086/304855.

\section{Permanent link}

http://nrs.harvard.edu/urn-3:HUL.InstRepos:41393139

\section{Terms of Use}

This article was downloaded from Harvard University's DASH repository, and is made available under the terms and conditions applicable to Other Posted Material, as set forth at http:// nrs.harvard.edu/urn-3:HUL.InstRepos:dash.current.terms-of-use\#LAA

\section{Share Your Story}

The Harvard community has made this article openly available.

Please share how this access benefits you. Submit a story.

Accessibility 


\title{
Microlensing of an Elliptical Source by a Point Mass
}

\author{
David Heyrovský and Abraham Loeb \\ Department of Astronomy, Harvard University, 60 Garden St., Cambridge, MA 02138
}

\begin{abstract}
We present an efficient method for computing lightcurves of an elliptical source which is microlensed by a point mass. The amplification of an extended source involves a two-dimensional integral over its surface brightness distribution. We show that for a general surface brightness profile with an elliptical symmetry, this integral can be reduced to one dimension. We derive analytical results for the entire lightcurve in the limit of low (e.g. planetary) mass lenses, and for the wings of all microlensing lightcurves in general. In both cases the lightcurve carries information about deviations of the source from elliptical symmetry, e.g. due to spots. The method is used to find the amplification of a circular red giant photosphere and an inclined accretion disk. We demonstrate that microlensing of an emission line from a disk can be used to infer the disk velocity structure and surface brightness profile.
\end{abstract}

Subject headings: gravitational lensing

To appear in The Astrophysical Journal, 1997

\section{Introduction}

The sample of all known gravitational lenses is by now dominated by stellar microlenses in our galaxy (Alcock et al. 1995, 1996; Udalski et al. 1994; Ansari et al. 1996; Alard 1996). Even galactic macro-lenses of quasars are often accompanied by microlensing events due to stars (Irwin et al. 1989; Schild and Smith 1991; Schneider, Ehlers, \& Falco 1992 and references therein), and thus emphasize that microlensing is a common phenomenon. Based on the fact that a solar mass lens at a distance $d$ offers an angular resolution $\sim(d / 10 \mathrm{Gpc})^{-1 / 2}$ micro-arcsec, microlensing has long been recognized as a potential tool for resolving the surface brightness distribution of compact sources.

Nevertheless, most stellar sources are smaller than the effective size of their lenses. The finite extent of a stellar photosphere $\left(\sim R_{\odot}\right)$ is still much smaller than the Einstein radius of a solar mass lens $\left[\sim 10^{3} R_{\odot} \times\left(M_{\text {lens }} / M_{\odot}\right)^{1 / 2}(d / 10 \mathrm{kpc})^{1 / 2}\right.$, where $M_{\text {lens }}$ is the lens mass $]$, and so the lightcurve of galactic microlenses typically carries little information about the brightness distribution of the source (Paczyński 1986; Udalski et al. 1994). The point source approximation breaks down only 
when the projected impact parameter is comparable to the finite size of the source. Although such circumstances are realized in a minority of all microlensing events, they carry a large scientific payoff. A spectroscopic identification of the source size and distance in such a case, could break the parameter degeneracy of the microlensing lightcurve and reveal the Einstein angle of the lens (Loeb \& Sasselov 1995; Gould \& Welch 1996). The appearance of extended source signatures should be common when giant stars are microlensed by planets (Loeb \& Sasselov 1995; Bennett \& Rhie 1996). By now, there are some examples of lightcurves with unusually high amplification (i.e. small impact parameter) or short duration (i.e. low lens masses) in which there are hints of finite source signatures (Mao et al. 1994; Lennon et al. 1996; Bennett et al. 1996; Pratt 1996; Alcock et al. 1997). Searches for microlensing by planets (Albrow et al. 1996; Pratt et al. 1996) are likely to add more examples to this class of events in the future. Spectroscopic monitoring of finite source events could ultimately test the theory of stellar photospheres, especially for giant stars (Loeb \& Sasselov 1995; Sasselov 1996).

An analogous ordering of scales appears in stellar microlensing of the continuum emission from quasars. For a star of mass $M_{\text {lens }}$, the Einstein radius at a cosmological distance is of order $\sim 5 \times 10^{16}\left(M_{\text {lens }} / M_{\odot}\right)^{1 / 2} \mathrm{~cm}$. In comparison, the optical continuum emission in quasars is believed to originate from a compact accretion disk. The UV bump observed in quasar spectra is often interpreted as thermal emission at a disk temperature $T_{\text {disk }} \sim 10^{5} T_{5} \mathrm{~K}$ (e.g. Laor 1990), and so the scale of the disk emission region must be $\sim 10^{15} T_{5}^{-2} L_{46}^{1 / 2} \mathrm{~cm}$, where $L_{46}$ is the corresponding luminosity of the quasar in units of $10^{46} \mathrm{erg} \mathrm{s}^{-1}$. Thus, for lens masses $M_{\text {lens }} \gg 10^{-3} M_{\odot}$, the continuum source is much smaller than the projected Einstein radius of the lens. This expectation is indeed confirmed in the lenses of Q2237+0305 (Irwin et al. 1989) and 0957+561 (Schild \& Smith 1991), where variability due to microlensing has been detected (Wambsganss, Paczyński, \& Schneider 1990; Rauch \& Blandford 1991; Racine 1991; Gould \& Miralda-Escudé 1996).

The above ordering of scales is reversed for the broad emission line regions of quasars. Reverberation studies of the time lag between variations in the continuum and the line emission in active galactic nuclei (AGN) indicate that the broad emission lines originate at a distance of $\sim 3 \times 10^{17} L_{46}^{1 / 2} \mathrm{~cm}$ (Peterson 1993; Maoz 1996). This implies that the broad lines could be amplified through stellar microlensing only for low-luminosity AGN $\left(L_{46} \lesssim 10^{-2}\right)$. Microlensing of Seyfert galaxies offers a unique opportunity for resolving the unknown structure and velocity distribution of the broad line region in AGN.

For all of the above applications, it is important to find the amplification in a variety of source and lens geometries. Evaluating the net amplification of an extended source involves a two-dimensional integral over its surface brightness distribution. A direct calculation of this integral raises some numerical problems (due to the divergence of the amplification at the lens position) and is computationally time consuming. Witt \& Mao (1994) expressed the amplification of a circular source with uniform brightness in terms of elliptic integrals. Alternatively, the amplification of uniform sources can be computed in the image plane, avoiding the divergence. Realistic sources, however, have a non-uniform surface brightness distribution (e.g. due to 
limb-darkening in stellar atmospheres) that also depends on the observed wavelength (Loeb \& Sasselov 1995; Gould \& Welch 1996; Sasselov 1996). Witt \& Mao (1994) proposed to sum over rings using the derivative of the brightness distribution as the appropriate weight for their contributions to the overall amplification. As a result, they traded one dimension in their surface integration for the evaluation of their elliptic integral solution. Other methods, such as image plane integration, require approximating nonuniform sources as a superposition of uniform sources. In this work, we propose another, more efficient approach to microlensing of nonuniform sources. We show how to analytically reduce the surface integral to one dimension for elliptically symmetric brightness profiles. This generalization can therefore be used to calculate microlensing of inclined disks, in addition to its straightforward application to stellar photospheres (Heyrovský, Loeb, \& Sasselov 1996).

Microlensing is also a valuable tool for studying sources with organized internal motions, such as rotating disks. If the gas in the disk emits a spectral line, then the variation of the line profile during a microlensing event would contain important information about the trajectory of the lens and the inclination angle, velocity structure, and brightness profile of the disk. As the lens moves across the disk, it amplifies the intensity at the Doppler shifted wavelength associated with the rotation velocity behind the lens. By examining the time dependence of the line profile it is possible, in principle, to deconvolve the radial brightness profile of the disk down to the innermost radius sampled by the lens trajectory. Examples for relevant sources include the iron K $\alpha$ fluorescence line in AGN (Tanaka et al. 1995; Fabian et al. 1995; Iwasawa et al. 1996), the maser lines emitted from compact disks in galactic nuclei (e.g., Miyoshi et al. 1995), and the line emission from stellar accretion disks (Warner 1995, and references therein). A related effect has previously been discussed in the context of the broad emission lines in AGN (Nemiroff 1988; Wambsganss 1990; Schneider \& Wambsganss 1990), although these lines are not believed to originate from a cold Keplerian disk because they do not posses a double-peaked profile.

In this work, we restrict our attention to microlensing by an isolated point mass lens, and avoid more complex lensing environments (see, e.g. Jaroszyński, Wambsganss, \& Paczyński 1992; Wambsganss \& Kundic 1995).

The outline of the paper is as follows. In $\S 2$ we derive our main mathematical results for the amplification of an elliptical source. In $\S 3$, we derive analytical expressions for the entire lightcurve of a low mass (e.g. planetary) lensing event (§3.1) and for the wings of the lightcurve in general (§3.2). Sections 4 and 5 are dedicated to specific astrophysical sources. In $\S 4$ we calculate sample lightcurves for a giant star with a realistic limb-darkened profile and for an inclined accretion disk. In $\S 5$ we describe the time dependence of emission line profiles from a Keplerian disk during a microlensing event. Finally, $\S 6$ summarizes the main conclusions of this work. 


\section{The Amplification Integral for Elliptical Sources}

In analysing the lensing geometry we use the two-dimensional sky coordinates. In this coordinate system, all projected distances are angular.

We consider a general elliptical source with eccentricity $e$ and semimajor axis $a$, which we set as our distance unit. The location of the lens at a given time can be defined by its displacement from the source center $\tau_{0}$ and by the angle $\alpha_{0}$ between the source-lens vector and the major axis of the source. Figure 1 depicts the geometry associated with our notations. A lens with an Einstein radius $\epsilon$ amplifies the flux from a point source at a distance $\tau$ by a factor

$$
A_{0}(\tau)=\frac{\tau^{2}+2 \epsilon^{2}}{\tau \sqrt{\tau^{2}+4 \epsilon^{2}}}
$$

In the limit of geometric optics, an extended source can be viewed as a sum of infinitesimal point-like sources. Its amplified flux can therefore be obtained by weighting $A_{0}(\tau)$ with the surface brightness distribution $B(\vec{r})$ and integrating over the projected surface area of the source $\Sigma_{S}$,

$$
F=a^{2} \int_{\Sigma_{S}} B(\vec{r}) \frac{\tau^{2}+2 \epsilon^{2}}{\tau \sqrt{\tau^{2}+4 \epsilon^{2}}} d \Sigma
$$

The net amplification is the ratio between this flux and the flux of the source in the absence of the lens,

$$
A \equiv F / F_{0},
$$

where

$$
F_{0}=a^{2} \int_{\Sigma_{S}} B(\vec{r}) d \Sigma
$$

It is most convenient to describe the surface brightness distribution of the source in terms of a coordinate system which is centered on the source. We set the $x$-axis along the major axis of the

ellipse (see Fig. 1), and adopt the following coordinates: $x=\rho \cos \psi, y=\rho \sqrt{1-e^{2}} \sin \psi$. Curves of constant $\rho$ are nested ellipses with semimajor axes $\rho$ and equal eccentricity $e ; \rho=1$ corresponds to the limb of the source. Note that $\psi$, which runs from 0 to $2 \pi$, is not the polar angle in the case of nonzero eccentricity. The area element is $d \Sigma=\sqrt{1-e^{2}} \rho d \rho d \psi$, and so equation (4) becomes

$$
F_{0}=a^{2} \sqrt{1-e^{2}} \int_{0}^{2 \pi} \int_{0}^{1} B(\rho, \psi) \rho d \rho d \psi
$$

In calculating the lensed flux in equation (2), it is advantageous to use lens-centered polar coordinates for which the amplification factor preserves its simple form (1). If we erect the corresponding $x_{1}$ axis from the lens towards the source center, we obtain the coordinates: $x_{1}=\tau \cos \phi, y_{1}=\tau \sin \phi$ (see Fig. 1). To express the source boundary and brightness distribution 
in terms of $\tau$ and $\phi$, we need to transform the source-centered coordinates to the lens-centered coordinates. As seen from Figure 1,

$$
\left(\begin{array}{l}
x \\
y
\end{array}\right)=\left(\begin{array}{cc}
-\cos \alpha_{0} & \sin \alpha_{0} \\
\sin \alpha_{0} & \cos \alpha_{0}
\end{array}\right)\left(\begin{array}{c}
x_{1}-\tau_{0} \\
y_{1}
\end{array}\right),
$$

or equivalently,

$$
\begin{aligned}
\rho \cos \psi & =-\tau \cos \left(\phi+\alpha_{0}\right)+\tau_{0} \cos \alpha_{0} \\
\rho \sqrt{1-e^{2}} \sin \psi & =\tau \sin \left(\phi+\alpha_{0}\right)-\tau_{0} \sin \alpha_{0} .
\end{aligned}
$$

From these equations it is straightforward to express both $\rho$ and $\psi$ in terms of $\tau$ and $\phi$. For $\rho$ we get

$$
\rho=\sqrt{\omega_{1}(\phi) \tau^{2}-\omega_{2}(\phi) \tau+\sigma_{0}^{2}}
$$

with

$$
\begin{aligned}
\omega_{1}(\phi) & =1+\frac{e^{2}}{1-e^{2}} \sin ^{2}\left(\phi+\alpha_{0}\right) \\
\omega_{2}(\phi) & =2 \tau_{0}\left[\cos \phi+\frac{e^{2}}{1-e^{2}} \sin \alpha_{0} \sin \left(\phi+\alpha_{0}\right)\right] \\
\sigma_{0} & =\tau_{0} \sqrt{1+\frac{e^{2}}{1-e^{2}} \sin ^{2} \alpha_{0}} .
\end{aligned}
$$

The parameter $\sigma_{0}$ is the value of $\rho$ at the position of the lens $(\tau=0)$. If the lens lies within the source boundary then $\sigma_{0}<1$, and if it lies beyond the boundary, $\sigma_{0}>1$. For a given angle $\phi$, the integration limits in the $\tau$ direction are found by setting $\rho=1$ in equation (8),

$$
\tau_{ \pm}(\phi)=\frac{\omega_{2} \pm \sqrt{\omega_{2}^{2}+4 \omega_{1}\left(1-\sigma_{0}^{2}\right)}}{2 \omega_{1}} .
$$

A line originating from the lens at an angle $\phi$ enters the source boundary at $\tau_{-}(\phi)$ (provided $\left.\sigma_{0}>1\right)$ and exits at $\tau_{+}(\phi)$. If $\sigma_{0}<1, \phi$ runs from $-\pi$ to $\pi$. To determine the $\phi$ integration limits in the $\sigma_{0}>1$ case, we set $\tau_{+}\left(\phi_{\lim }\right)=\tau_{-}\left(\phi_{\text {lim }}\right)$ in equation (10). After some algebra we find,

$$
\begin{aligned}
& \left(\begin{array}{c}
\cos 2 \phi_{\lim } \\
\sin 2 \phi_{\lim }
\end{array}\right)\left(\tau_{0}^{4}+e^{4}-2 e^{2} \tau_{0}^{2} \cos 2 \alpha_{0}\right) \\
& =\left(\tau_{0}^{2}+e^{2}-2\right)\left(\begin{array}{c}
\tau_{0}^{2}-e^{2} \cos 2 \alpha_{0} \\
e^{2} \sin 2 \alpha_{0}
\end{array}\right) \pm 2 \sqrt{\tau_{0}^{2}+e^{2}-e^{2} \tau_{0}^{2} \cos ^{2} \alpha_{0}-1}\left(\begin{array}{c}
-e^{2} \sin 2 \alpha_{0} \\
\tau_{0}^{2}-e^{2} \cos 2 \alpha_{0}
\end{array}\right) .
\end{aligned}
$$

This expression generally yields four different solutions for $\phi_{\lim }$, due to the implicit $\pm \pi$ ambiguity in this angle. The two values of interest are directed towards the source, and for them $\omega_{2}\left(\phi_{\lim }\right) \geq 0$. We use $\phi_{-} \in(-\pi, 0)$ as the lower limit, and $\phi_{+} \in(0, \pi)$ as the upper limit. Since the area element 
in the lens-centered coordinates is $d \Sigma=\tau d \tau d \phi$, the divergence of the integrand in equation (2) at $\tau=0$ is eliminated. The explicit form of equation (2) as a function of the lens position is:

$$
F\left(\tau_{0}, \alpha_{0}\right)=a^{2} \int_{-\pi}^{\pi} \int_{0}^{\tau_{+}(\phi)} B(\rho, \psi) \frac{\tau^{2}+2 \epsilon^{2}}{\sqrt{\tau^{2}+4 \epsilon^{2}}} d \tau d \phi \quad, \quad \text { for } \quad \sigma_{0}<1
$$

and

$$
F\left(\tau_{0}, \alpha_{0}\right)=a^{2} \int_{\phi_{-} \tau_{-}(\phi)}^{\phi_{+}} \int_{\tau_{+}(\phi)} B(\rho, \psi) \frac{\tau^{2}+2 \epsilon^{2}}{\sqrt{\tau^{2}+4 \epsilon^{2}}} d \tau d \phi \quad, \quad \text { for } \quad \sigma_{0}>1
$$

So far we made no assumptions about the brightness distribution $B(\rho, \psi)$. In the following we concentrate on elliptical source profiles with $B$ being independent of $\psi$. In particular, if the brightness can be written as a series in powers of $\rho^{2}$

$$
B(\rho)=\sum_{i=0}^{k} \beta_{i} \rho^{2 i}
$$

then equation (8) can be used to transform this series to a polynomial in $\tau$,

$$
B(\tau, \phi)=\sum_{L=0}^{k} \sum_{N=0}^{k-L} \frac{\omega_{1}^{L}\left(-\omega_{2}\right)^{N}}{L ! N !} B^{(L+N)} \tau^{2 L+N} .
$$

Here the constants $B^{(n)}$ are the $n$-th derivatives of the brightness profile (14) with respect to $\rho^{2}$ at the position of the lens,

$$
B^{(n)}=\left[\frac{d^{n} B}{d\left(\rho^{2}\right)^{n}}\right]_{\rho=\sigma_{0}}=\sum_{i=n}^{k} \frac{i !}{(i-n) !} \beta_{i} \sigma_{0}^{2(i-n)} .
$$

Note that in this expression the derivatives of the polynomial (14) are formally evaluated at the lens position even if it lies beyond the source boundary.

By substituting expression (15) into equations (12) and (13), we are left with $\tau$-integrals of the general form

$$
J_{h}(\mu, \nu)=\int_{\mu}^{\nu} \tau^{h} \frac{2 \tau^{2}+4 \epsilon^{2}}{\sqrt{\tau^{2}+4 \epsilon^{2}}} d \tau
$$

where $h=0, \ldots, 2 k$. These integrals can be solved analytically. We get

$$
J_{0}(\mu, \nu)=\left[\tau \sqrt{\tau^{2}+4 \epsilon^{2}}\right]_{\mu}^{\nu} ;
$$

for $p \geq 0$

$$
J_{2 p+1}(\mu, \nu)=\frac{1}{2 p+3}\left[\sqrt{\tau^{2}+4 \epsilon^{2}}\left\{2 \tau^{2 p+2}-\frac{4 \epsilon^{2}}{\left(\begin{array}{c}
2 p \\
p
\end{array}\right)} \sum_{i=0}^{p}\left(\begin{array}{c}
2 i \\
i
\end{array}\right)\left(-16 \epsilon^{2}\right)^{p-i} \tau^{2 i}\right\}\right]_{\mu}^{\nu}
$$


and for $p>0$

$$
J_{2 p}(\mu, \nu)=\frac{1}{p+1}\left[\tau^{2 p+1} \sqrt{\tau^{2}+4 \epsilon^{2}}+4 p\left(\begin{array}{c}
2 p \\
p
\end{array}\right)\left(-\epsilon^{2}\right)^{p+1}\left\{\operatorname{arcsh} \frac{\tau}{2 \epsilon}+\sqrt{\tau^{2}+4 \epsilon^{2}} \sum_{i=1}^{p} \frac{\left(-\epsilon^{2}\right)^{-i}}{2 i\left(\begin{array}{c}
2 i \\
i
\end{array}\right)} \tau^{2 i-1}\right\}\right]_{\mu}^{\nu} .
$$

In this way the two-dimensional integration over the source is reduced to a $\phi$-integral. Equations (12) and (13) now read

$$
F\left(\tau_{0}, \alpha_{0}\right)=\frac{a^{2}}{2} \int_{-\pi}^{\pi} \sum_{L=0}^{k} \sum_{N=0}^{k-L} \frac{\omega_{1}^{L}(\phi)\left[-\omega_{2}(\phi)\right]^{N}}{L ! N !} B^{(L+N)} J_{2 L+N}\left(0, \tau_{+}(\phi)\right) d \phi, \quad \text { for } \quad \sigma_{0}<1,
$$

and

$$
F\left(\tau_{0}, \alpha_{0}\right)=\frac{a^{2}}{2} \int_{\phi_{-}}^{\phi_{+}} \sum_{L=0}^{k} \sum_{N=0}^{k-L} \frac{\omega_{1}^{L}(\phi)\left[-\omega_{2}(\phi)\right]^{N}}{L ! N !} B^{(L+N)} J_{2 L+N}\left(\tau_{-}(\phi), \tau_{+}(\phi)\right) d \phi, \quad \text { for } \quad \sigma_{0}>1 .
$$

For completeness, we substitute equation (14) into equation (5) to get the unlensed flux

$$
F_{0}=\pi a^{2} \sqrt{1-e^{2}} \sum_{i=0}^{k} \frac{\beta_{i}}{i+1}
$$

Surface brightness profiles of astrophysical sources are often defined through a set of discrete points $\left[\rho_{i}, B_{i}\right], i=1, \ldots, n$, with $\rho_{1}=0$ and $\rho_{n}=1$. A straightforward application of the above results could be obtained through a least-squares fit of the form (14) to the data points. However, a satisfactory global fit for $\rho \in\langle 0,1\rangle$ often requires the use of many high-order terms in the series. For the $k+1$ terms of equation (14) it is necessary to evaluate $2 k+1$ functions $J_{0} \cdots J_{2 k}$, with increasing complexity at increasing $k$. As a result, an appropriate numerical integration scheme (such as Gaussian quadrature) would be more effective for high $k$. An alternative possibility is to interpolate between pairs of neighboring data points, so that for $\rho \in\left\langle\rho_{i}, \rho_{i+1}\right\rangle$ the brightness is described by an adequate function $B_{i}(\rho)$. A simple example for an interpolation scheme involves

$$
B_{i}(\rho)=a_{i}+b_{i} \rho^{2}
$$

where $a_{i}$ and $b_{i}$ are determined by the continuity conditions at the boundary points,

$$
B_{i}\left(\rho_{i}\right)=B_{i}, \quad B_{i}\left(\rho_{i+1}\right)=B_{i+1}, \quad i=1 \ldots n-1 .
$$

This interpolation scheme for the brightness profile does not have a continuous derivative at the data points $\rho_{i}$. Smoothness can be easily achieved by adding a $\rho^{4}$ term to equation (24) and by adding conditions for the continuity of the derivative in equation (25).

Following the interpolation scheme of equation (24), the source is divided into $n-1$ concentric bands, bounded by ellipses with semimajor axes $\rho_{i}$. The brightness profile inside the $j$-th band is 
described by values $a_{j}$ and $b_{j}$. A line of constant angle $\phi$ from the lens passes through a sequence of $l$ bands in the $\tau$-direction, and crosses their boundaries at values $\tau_{1}<\tau_{2}<\cdots<\tau_{l+1}$ (see Fig. 2 ). We correspondingly substitute in the integrand of equation (21)

$$
B^{(L+N)} J_{2 L+N}\left(0, \tau_{+}\right) \longrightarrow \sum_{i=1}^{l} B_{s_{i}}^{(L+N)} J_{2 L+N}\left(\tau_{i}, \tau_{i+1}\right)
$$

where $\tau_{1}=0, \tau_{l+1}=\tau_{+}$, and $s_{i}$ is the index of the band crossed between $\tau_{i}$ and $\tau_{i+1}$. An analogous substitution with $\tau_{1}=\tau_{-}$should be performed in the integrand of equation (22).

The sequence of bands that are crossed can easily be determined from the geometry illustrated in Figure 2. If the lens lies outside the source boundary $\left(\sigma_{0}>1\right)$, one needs to find the innermost band which is crossed. The $\rho$-interval of this band contains the minimum value of $\rho$ which is reached along the $\phi=$ const ray. Equation (8) yields

$$
\rho_{\min }=\sqrt{\sigma_{0}^{2}-\frac{\omega_{2}^{2}}{4 \omega_{1}}} .
$$

If the lens lies inside the source boundary $\left(\sigma_{0}<1\right)$, the sequence of bands starts at the lens position $\left(\rho=\sigma_{0}\right)$, and the band with $\rho_{\min }$ has to be determined only if $\omega_{2}>0$. To calculate the crossing points $\tau_{i}$, it is then sufficient to know that intersections with ellipse $\rho_{j}$ occur at

$$
\tau=\frac{\omega_{2} \pm \sqrt{\omega_{2}^{2}+4 \omega_{1}\left(\rho_{j}^{2}-\sigma_{0}^{2}\right)}}{2 \omega_{1}} .
$$

If the necessary number of bands is high, numerical evaluation of the $\tau$ integral should be considered as an alternative.

The method has so far been presented for an elliptical source. Most of the equations are considerably simpler for a circular source. By setting the eccentricity to zero, $e=0$, the source-centered coordinates $\rho, \psi$ become regular polar coordinates, with $a$ being the source radius. Equations (9) reduce to: $\omega_{1}=1, \omega_{2}(\phi)=2 \tau_{0} \cos \phi$, and $\sigma_{0}=\tau_{0}$. The $\tau$ integration limits in equation (10) are

$$
\tau_{ \pm}=\tau_{0} \cos \phi \pm \sqrt{1-\tau_{0}^{2} \sin ^{2} \phi}
$$

and the angular integration limits in equation (11) are given by

$$
\phi_{ \pm}= \pm \arcsin \frac{1}{\tau_{0}}
$$

Due to the circular symmetry, the angular integration in equations (21) and (22) can be performed over half the interval and the result multiplied by a factor of two.

In principle, our method can also be used to describe the effect of spots on lightcurves of extended sources (Sasselov 1996). Since the observed flux is linearly dependent on the source brightness, one could simply superimpose the lightcurve of an elliptical spot on top of the background lightcurve. 


\section{Analytical Results}

\subsection{Low Mass Lenses}

The results obtained for brightness profile (14) can be used to study the limiting case when the Einstein radius of the lens is much smaller than the source size $(\epsilon \ll 1)$. The lensed flux in equations (21) and (22) depends on $\epsilon$ only through the functions $J_{h}$ in equations (18)-(20). For small $\epsilon$, these functions can be expanded as power series in $\epsilon$. We make use of the expansions

$$
\begin{aligned}
\sqrt{\tau^{2}+4 \epsilon^{2}} & =\tau+\frac{2 \epsilon^{2}}{\tau}-\frac{2 \epsilon^{4}}{\tau^{3}}+O\left(\epsilon^{6}\right) \\
\operatorname{arcsh} \frac{\tau}{2 \epsilon} & =-\ln \epsilon+\ln \tau+\frac{\epsilon^{2}}{\tau^{2}}+O\left(\epsilon^{4}\right) .
\end{aligned}
$$

If the lens position is inside the source boundary $\left(\sigma_{0}<1\right)$, we obtain

$$
\begin{aligned}
J_{0}\left(0, \tau_{+}\right) & =\tau_{+}^{2}+2 \epsilon^{2}-\frac{2 \epsilon^{4}}{\tau_{+}^{2}}+o\left(\epsilon^{4}\right) \\
J_{1}\left(0, \tau_{+}\right) & =\frac{2}{3} \tau_{+}^{3}+\frac{8 \epsilon^{3}}{3}-\frac{4 \epsilon^{4}}{\tau_{+}}+o\left(\epsilon^{4}\right) \\
J_{2}\left(0, \tau_{+}\right) & =\frac{\tau_{+}^{4}}{2}-4 \epsilon^{4} \ln \epsilon+\left(4 \ln \tau_{+}-3\right) \epsilon^{4}+o\left(\epsilon^{4}\right) \\
L>2 \quad J_{L}\left(0, \tau_{+}\right) & =\frac{2}{L+2} \tau_{+}^{L+2}+\frac{4 \epsilon^{4}}{L-2} \tau_{+}^{L-2}+o\left(\epsilon^{4}\right) .
\end{aligned}
$$

If the lens is outside the source $\left(\sigma_{0}>1\right)$, we get

$$
\begin{aligned}
L \neq 2 \quad J_{L}\left(\tau_{-}, \tau_{+}\right) & =\left[\frac{2}{L+2} \tau^{L+2}+\frac{4 \epsilon^{4}}{L-2} \tau^{L-2}\right]_{\tau_{-}}^{\tau_{+}}+o\left(\epsilon^{4}\right) \\
J_{2}\left(\tau_{-}, \tau_{+}\right) & =\left[\frac{\tau^{4}}{2}+4 \epsilon^{4} \ln \tau\right]_{\tau_{-}}^{\tau_{+}}+o\left(\epsilon^{4}\right)
\end{aligned}
$$

The observed flux can now be obtained from equations (21) and (22):

$$
F\left(\tau_{0}, \alpha_{0}\right)=F_{0}+2 \pi a^{2} \epsilon^{2} B^{(0)}+O\left(\epsilon^{4} \ln \epsilon\right) \quad \text { for } \sigma_{0}<1,
$$

and

$$
F\left(\tau_{0}, \alpha_{0}\right)=F_{0}+2 \epsilon^{4} a^{2} \int_{\Sigma} \frac{B}{\tau^{4}} d \Sigma+O\left(\epsilon^{6}\right) \quad \text { for } \sigma_{0}>1
$$

Since $B^{(0)}$ is the brightness at the position of the lens, the lightcurve traces the brightness profile along the lens trajectory to leading order $\left(\epsilon^{2}\right)$. For $\sigma_{0}<1$, the excess flux equals twice the Einstein ring area times $B^{(0)}$. The accuracy of this leading-order approximation will be demonstrated through a numerical example in $\S 4$. We expect the first two terms in equations (34) 
and (35) to hold in general for an arbitrary surface brightness distribution, as long as the variations in $B(\vec{r})$ are weak on the scale of the Einstein radius of the lens. Note that the convergence of the above expansions requires that $2 \epsilon$ be smaller than $\tau_{-}$and $\tau_{+}$. Hence, the derived expression for the lightcurve is valid as long as the lens lies a distance greater than $2 \epsilon$ away from the source boundary.

\subsection{Lightcurve Wings}

We next study the effect that a finite source size has on the wings of a general lightcurve. For this purpose we start from equation (2) and place no restrictions on the brightness distribution $B(\vec{r})$. We associate the "wings" of the lightcurve with the region over which the lens-source separation is larger than both the Einstein diameter of the lens and the size of the source, i.e. we assume that $\tau_{0} \gg 2 \epsilon$ and $\tau_{0} \gg 1$. Rewriting equation (2) in terms of source coordinates, we get

$$
F=a^{2} \sqrt{1-e^{2}} \int_{0}^{2 \pi} \int_{0}^{1} B(\rho, \psi)\left(1+\frac{2 \epsilon^{2}}{\tau^{2}}\right)\left(1+\frac{4 \epsilon^{2}}{\tau^{2}}\right)^{-\frac{1}{2}} \rho d \rho d \psi
$$

where $\tau^{2}$ can be expressed from equations (7),

$$
\tau^{2}=\tau_{0}^{2}-2 \rho \tau_{0}\left(\cos \alpha_{0} \cos \psi-\sqrt{1-e^{2}} \sin \alpha_{0} \sin \psi\right)+\rho^{2}\left(1-e^{2} \sin ^{2} \psi\right)
$$

The amplification factor in the integrand of equation (36) can be expanded in powers of $\tau_{0}^{-1}$,

$$
\left(1+\frac{2 \epsilon^{2}}{\tau^{2}}\right)\left(1+\frac{4 \epsilon^{2}}{\tau^{2}}\right)^{-\frac{1}{2}}=1+\frac{2 \epsilon^{4}}{\tau_{0}^{4}}+\frac{8 \rho \epsilon^{4}}{\tau_{0}^{5}}\left(\cos \alpha_{0} \cos \psi-\sqrt{1-e^{2}} \sin \alpha_{0} \sin \psi\right)+O\left(\tau_{0}^{-6}\right) .
$$

By substituting this result into equation (36) we obtain,

$$
F=\left(1+\frac{2 \epsilon^{4}}{\tau_{0}^{4}}\right) F_{0}+\frac{8 \epsilon^{4} a^{2} \sqrt{1-e^{2}}}{\tau_{0}^{5}} \int_{0}^{1} \int_{0}^{2 \pi} B\left(\cos \alpha_{0} \cos \psi-\sqrt{1-e^{2}} \sin \alpha_{0} \sin \psi\right) \rho^{2} d \psi d \rho+O\left(\tau_{0}^{-6}\right) .
$$

The leading correction to the unlensed flux (of order $\tau_{0}^{-4}$ ) is independent of the source structure, and appears also for a point source. The next term (of order $\tau_{0}^{-5}$ ) vanishes for a point source, as well as for any symmetric source with $B(\rho, \psi)=B(\rho)$. The integral in this term is, in fact, the projection of the "brightness dipole moment" $\int \vec{r} B(\vec{r}) d \Sigma$ on the source-lens direction. This term therefore reflects the asymmetry in the brightness distribution of the source, which could result from the existence of hot or cold spots on its surface (Sasselov 1996; Gould \& Miralda-Escudé 1996). The term vanishes if we redefine the positions relative to the center of brightness, rather than the geometrical center of the source. 


\section{Sample Lightcurves of Astrophysical Sources}

The microlensing lightcurve of the source is obtained from the time dependence of the lens position coordinates, $\tau_{0}$ and $\alpha_{0}$. A linear motion of the lens with respect to the source can be described by two parameters: the angle $\beta$ between the major axis of the source and the lens trajectory, and the impact parameter $p$ (see Fig. 3). To avoid ambiguity, we assign a negative sign to $p$ if the source center lies to the right of the lens trajectory. For convenience, we normalize the time $t$ in units of semimajor axis crossing-time and set $t=0$ at closest approach. The geometry illustrated in Figure 3 then yields

$$
\begin{aligned}
\tau_{0} & =\sqrt{p^{2}+t^{2}} \\
\left(\begin{array}{c}
\cos \alpha_{0} \\
\sin \alpha_{0}
\end{array}\right) & =\frac{1}{\tau_{0}}\left(\begin{array}{cc}
\cos \beta & \sin \beta \\
-\sin \beta & \cos \beta
\end{array}\right)\left(\begin{array}{l}
t \\
p
\end{array}\right) .
\end{aligned}
$$

In the case of a circular source (e.g. a lensed star), only $p$ needs to be specified and the angles $\beta$ and $\alpha_{0}$ are redundant.

As an example of stellar microlensing we present a theoretical lightcurve for the MACHO 95-30 event (Pratt 1996, Alcock et al. 1997). The source, a red giant in the galactic bulge, was amplified by a lens with an Einstein radius $\epsilon \approx 13$ and an impact parameter $p \approx 0.7$. The $\mathrm{R}$-band brightness profile shown in Figure 4 is based on an atmosphere model calculation of the source by Sasselov (1996). The computed lightcurve is plotted in Figure 5 together with a point-source lightcurve, for comparison.

Figure 6 compares the exact lightcurve of a low mass lens with the leading order term in the expansion described by equation (34). In difference from the real MACHO 95-30 event, we assume in this example that the lens has an Einstein radius $\epsilon=0.1$ and an impact parameter $p=0.2$. In this case, the leading order term (of order $\epsilon^{2}$ ) in equation (34) captures the main characteristics of the lightcurve to a reasonable accuracy.

The case of an elliptical source can be illustrated through the example of microlensing of an AGN disk. A circular disk which is inclined by an angle $i$ relative to the line-of-sight, would appear as an ellipse with eccentricity $e=\sin i$ on the sky projection. The semimajor axis would then equal the disk radius, and the $\rho=$ const contours would be elliptical projections of circles, with $\psi$ being the standard polar angle on the face of the disk. In calculating a sample lightcurve for this application, we use the brightness profile of a stationary thin accretion disk (see Frank, King, \&

Raine 1985) with $B(\rho) \propto\left[1-\sqrt{\rho_{i n} / \rho}\right] \rho^{-3}$. We assume a disk with an inner radius $\rho_{i n}=0.1$ and an inclination $i=60^{\circ}$, which is lensed by a lens with an Einstein radius $\epsilon=5$. Figure 7 contains lightcurves for events with the same impact parameter $p=0.8$ but different approach angles $\beta$. While the lightcurves of a circularly symmetric source are invariant to $\beta$, the inclination of the disk leaves a distinct $\beta$-dependent signature on the amplitude and symmetry of its lightcurve. 


\section{Microlensing of an Emission Line From a Keplerian Disk}

Next we consider the effect of microlensing on the spectral shape of an emission line from an accretion disk. Because of the Doppler shift associated with the disk rotation, the amplification as a function of wavelength could help unravel the inclination and surface brightness distribution of the disk.

To examine the microlensing distortion of the line profile we adopt the simplest model of a planar thin Keplerian disk with an inner radius $\rho_{i n}$ and inclination $i$. We parametrize the disk surface by the coordinates $\rho, \psi$ which were introduced in $\S 2$, and assume a Keplerian velocity

profile $v(\rho)=v_{i n} \sqrt{\rho_{i n} / \rho}$, where $v_{i n}$ is the velocity at the inner edge of the disk. We assume that the cold gas in the disk emits a line at a rest-frame wavelength $\lambda_{0}$, and with a thermal width that is much smaller than the Doppler width induced by the rotation of the disk. We therefore approximate the rest-frame line profile by a delta-function centered on $\lambda_{0}$. For simplicity, we neglect any relativistic effects on the radiation emitted by the disk. Due to variations of the line-of-sight velocity across the source, the Doppler-shifted wavelength reaching the observer from a point $(\rho, \psi)$ is

$$
\lambda=\lambda_{0}\left[1+\frac{v(\rho)}{c} \sin i \cos \psi\right],
$$

where $c$ is the speed of light. In this notation, $\psi=0$ is on the redshifted half of the disk. The spectral intensity profile of the line emitted by a microlensed disk is then

$$
I(\lambda)=\int_{\rho_{i n}}^{1} \int_{-\pi}^{\pi} j(\rho) \delta\left[\lambda-\lambda_{0}\left(1+\frac{v(\rho)}{c} \sin i \cos \psi\right)\right] A_{0}(\tau(\rho, \psi)) \rho \cos i d \psi d \rho
$$

where $j(\rho)$ is the spectral emissivity and $A_{0}(\tau)$ is the amplification factor in equation (11). The displacement $\tau(\rho, \psi)$ of the lens from the integration point is given by equation (37) with $e=\sin i$. We normalize the wavelength shift by its maximum value, $\left(v_{i n} / c\right) \sin i$, and denote the normalized fractional shift by $\Delta=\left(\lambda / \lambda_{0}-1\right) /\left[\left(v_{i n} / c\right) \sin i\right]$. Integration of the delta-function in equation (43) over $\psi$ yields

$I\left(\lambda_{0}\left[1+\Delta \frac{v_{i n}}{c} \sin i\right]\right)=\int_{\rho_{i n}}^{\rho_{\max }} j(\rho) \frac{A_{0}\left(\tau\left(\rho, \arccos \left[\Delta \sqrt{\rho / \rho_{i n}}\right]\right)\right)+A_{0}\left(\tau\left(\rho,-\arccos \left[\Delta \sqrt{\rho / \rho_{\text {in }}}\right]\right)\right)}{\lambda_{0}\left(v_{i n} / c\right) \sqrt{\rho_{i n} / \rho-\Delta^{2}} \tan i} \rho d \rho$.

The two terms in the numerator reflect the fact that points with angles $\psi$ and $-\psi$ have the same line-of-sight velocity. The upper integration limit depends on wavelength. For small wavelength shifts $|\Delta| \leq \sqrt{\rho_{i n}}$, one gets contributions from all radii so that $\rho_{\max }=1$. Larger shifts $|\Delta| \geq \sqrt{\rho_{i n}}$ originate only from the inner disk, with $\rho_{\max }=\rho_{i n} / \Delta^{2}$.

For concreteness, we assume a power-law profile for the disk emissivity, $j(\rho) \propto \rho^{n}$. Figure 8 shows the sensitivity of the unlensed line profile to the power-law index $n$. For the purpose of this illustration, we have kept the total luminosity of the disks constant, namely $\int j(\rho) \rho d \rho=$ const. 
The unlensed profiles show two characteristic peaks at $|\Delta|=\sqrt{\rho_{\text {in }}}$. We have chosen $\rho_{\text {in }}=0.01$ and $i=60^{\circ}$ in the examples shown. As is apparent, the more centrally concentrated the emissivity law is, the more pronounced the line wings are and the less pronounced the peaks are. This tendency results from the enhanced weight that the inner region of the disk obtains in the intensity integral (44), as $n$ gets more negative. The wings of the line correspond to high rotation speeds which are generically obtained in the inner region of the disk.

Figure 9 shows the effect of lensing on the line profile for $n=-1$. In this calculation we assumed a lens with an Einstein radius $\epsilon=1$, located at several different positions along the major axis of the source $\left(\alpha_{0}=0\right)$. For clarity, we show results only for the redshifted side of the disk. Apart from an overall amplification of the total flux in the line, lensing introduces an asymmetry between the intensities of the two peaks. The position of the peaks remains unaffected. The curve with $\tau_{0}=0.1$ illustrates another feature, present when the lens is positioned on top of the disk. In this situation, the profile diverges at the emission wavelength of the geometric point behind the lens, $\Delta=\sqrt{\rho_{\text {in }} / \rho_{\text {lens }}} \cos \psi_{\text {lens. }}$. This divergence is integrable, and so a filter with a finite wavelength resolution would register it as a third peak. In reality, the formal divergence will be smoothed out by the thermal width of the line. The time-dependent intensity profile around the moving third peak can be used to map the axisymmetric brightness profile of the disk as the lens scans the source along its linear trajectory.

The asymmetry of the microlensed line profiles in Figure 9 indicates a change in the mean redshift of the emitted line, depending on the lens position. This effect has been previously studied for various models of the broad line regions of quasars (Nemiroff 1988; Schneider \& Wambsganss 1990). The redshift change $\Delta z$ can be simply computed from the spectral intensity $I$ in equation (44) as

$$
\Delta z=\frac{\int_{-1}^{1} I \Delta d \Delta}{\int_{-1}^{1} I d \Delta} \frac{v_{i n}}{c} \sin i .
$$

Figure 10 shows the change in redshift as a function of lens position along the major axis of the disk, for the same situation as in Figure 9. In this case the redshift can change by more then $10 \%$ of the maximum Doppler shift from the inner edge of the disk, if the lens is positioned close to the inner edge.

\section{Discussion}

We have developed an efficient method for computing microlensing lightcurves of sources with an elliptical symmetry and a general surface brightness distribution. Equations (18)-(23) express the amplification of elliptical sources in terms of a one-dimensional integral. This integral is considerably simpler for a circular source. The method is well suited for the study of spectral changes due to microlensing. Such changes have already been observed in the MACHO 95-30 
event (Alcock et al. 1997). We are currently using the technique for modelling these observations (Sasselov, Heyrovský \& Loeb 1997). The method can also be easily extended to describe the effect of elliptical spots on the background lightcurve. We have derived fully analytical results in the limit of low lens masses [cf. equations (34), (35)] and for the wings of the lightcurve in general [equation (39)]. In both cases, the lightcurve carries information about deviations of the source from elliptical symmetry (e.g., due to hot or cold spots). The low mass limit case applies to lensing of giant stars in the Milky Way bulge by planets of mass $M_{\mathrm{pl}}$, for which the Einstein radius is $\sim 3 R_{\odot} \times\left(M_{\mathrm{pl}} / M_{\oplus}\right)^{1 / 2}$. The lightcurve in this case obtains the form of a low amplitude increase in the source intensity over the source crossing time. The amplitude of the excess flux is twice the area of the Einstein ring times the brightness of the source at the lens position [cf. equation (34)]. Events of this type might soon be discovered through ongoing microlensing searches for planets (Albrow et al. 1996; Pratt et al. 1996). Finite source signatures appear whenever the impact parameter is comparable to the source size, including high amplification events caused by massive lenses. Figure 5 shows the theoretical lightcurve corresponding to the source and lens parameters of a microlensing event of this type, MACHO 95-30 (Pratt 1996, Alcock et al. 1997). The detection rate of events with finite source signatures is expected to increase if future searches will focus on the source population of giant stars (Gould 1995a). When such signatures are combined with spectroscopic identification of the sources, they can be used to break the degeneracy of microlensing lightcurves (Loeb \& Sasselov 1995; Gould \& Welch 1996).

Our formalism is particularly suitable for the analysis of microlensing of inclined accretion disks which appear elliptical in projection on the sky. The microlensing lightcurve of the disk depends on its surface brightness distribution and inclination (cf. Fig. 7). If a thin disk emits a spectral line, then it is possible to deconvolve its properties from the variation of the line profile during a microlensing event. When the lens position overlaps with the disk, a third peak is added to the standard "double-peak" profile of the disk emission. The microlensing peak appears at the emission wavelength corresponding to the Doppler velocity behind the lens (cf. Fig. 9). As the lens moves along a straight line, the temporal evolution of the intensity and wavelength of the third peak can be used to map the disk structure. Thus, microlensing of a spectral line offers a unique opportunity for unraveling the surface brightness and velocity profiles of accretion disks. The main practical obstacle for such mappings is the potential for intrinsic variability in the disk properties during the event. Figure 10 illustrates the change in the mean redshift of the spectral line, due to the asymmetry of the microlensed profiles. The change can exceed $10 \%$ of the maximum Doppler shift in the disk.

The signatures described in this paper should be more complicated in environments where the microlensing optical depth is not small, such as the cores of galactic macro-lenses at cosmological distances. However, our isolated point lens calculation still applies to the outer halos of such galaxies, where the optical depth is low. Quasar absorption spectra can be used to select such foreground galactic halos. In particular, damped Ly $\alpha$ or metal-line absorption features are often associated with projected impact parameters of $\sim 10-100 \mathrm{kpc}$ from galactic centers (Steidel et al. 
1994, 1995, 1996), and could therefore be used to signal an intervening galactic halo. If galactic halos are composed of Massive Compact Halo Objects, then a significant fraction of all damped Ly $\alpha$ absorbers should show evidence for isolated microlensing events (Perna \& Loeb 1997), of the type discussed in this work. Isolated microlensing events from intergalactic stars are also possible but only for a minority of all quasars (Dalcanton et al. 1994; Gould 1995b).

We thank Dimitar Sasselov for providing the brightness profile of the MACHO 95-30 source, and Robert Nemiroff for suggesting the calculation of the change in the mean redshift of microlensed emission lines. We also thank both for valuable comments on the manuscript. This work was supported in part by the NASA ATP grant NAG5-3085 and the Harvard Milton fund (for AL).

\section{REFERENCES}

Alard, C. 1996, in Proc. IAU Symp. 173, Astrophysical Applications of Gravitational Lensing, p. 214, Eds. C. S. Kochanek \& J. N. Hewitt, (Dordrecht: Kluwer Academic Publishers)

Albrow, M., et al. 1996, in Proc. IAU Symp. 173, Astrophysical Applications of Gravitational Lensing, p. 227, Eds. C. S. Kochanek \& J. N. Hewitt, (Dordrecht: Kluwer Academic Publishers)

Alcock, C., et al. 1995, ApJ, submitted, preprint astro-ph/9512146

Alcock, C., et al. 1996, ApJ, submitted, preprint astro-ph/9606165

Alcock, C., et al. 1997, ApJ, submitted, preprint astro-ph/9702199

Ansari, R., et al. 1996, A\&A, 314, 94

Bennett, D., et al. 1996, preprint astro-ph/9612208

Bennett, D., \& Rhie, S. H. 1996, ApJ, 472, 660

Dalcanton, J. J., Canizares, C. R., Granados, A., Steidel, C. C., \& Stocke, J. T. 1994, ApJ, 424, 550

Fabian, A. C., Nandra, K., Reynolds, C. S., Brandt, W. N., Otani, C., Tanaka, Y., Inoue, H., \& Iwasawa, K. 1995, MNRAS, 277, L11

Frank, J., King, A., \& Raine, D. 1985, Accretion Power in Astrophysics (Cambridge: Cambridge Univ. Press)

Gould, A. 1995a, ApJ, 447, 491

Gould, A. 1995b, ApJ, 455, 37

Gould, A., \& Miralda-Escudé, J. 1996, preprint astro-ph/9612144

Gould, A., \& Welch, D. L. 1996, ApJ, 464, 212 
Heyrovský, D., Loeb, A., \& Sasselov, D. 1996, in Proc. IAP Colloquium 12, Variable Stars and the Astrophysical Returns of Microlensing Surveys, eds. R. Ferlet and J. P. Maillard, (Paris: Editions Frontieres)

Irwin, M. J., Webster, R. L., Hewett, P. C., Corrigan, R. T., \& Jedrzejewski, R. I. 1989, AJ, 98, 1989

Iwasawa, K., et al. 1996, MNRAS, 282, 1038

Jaroszyński, M., Wambsganss, J., \& Paczyński, B. 1992, ApJ, 396, L65

Laor, A. 1990, MNRAS, 246, 369

Lennon, D. J., Mao, S., Fuhrmann, K., \& Gehren, T. 1996, ApJ, 471, L23

Loeb, A., \& Sasselov, D. 1995, ApJ, 449, L33

Mao, S., et al. 1994, Bull. American Astron. Soc., 185, \#17.05

Maoz, D. 1996, to appear in the proceedings of IAU Colloquium 159, Shanghai, June 1996, preprint astro-ph/9609174

Maoz, D., \& Gould, A. 1994, ApJ, 425, L67

Miyoshi, M., Moran, J., Herrnstein, J., Greenhill, L., Nakai, N., Diamond, P., \& Inoue, M. 1995, Nature, 373, 127

Nemiroff, R. 1988, ApJ, 335, 593

Paczyński, B. 1986, ApJ, 304, 1

Perna, R., \& Loeb, A. 1997, ApJ, submitted, astro-ph/9701226

Peterson, B. M. 1993, PASP, 105, 247

Pratt, M. R. 1996, in collection of talks in the 2nd International Workshop on Gravitational Microlensing Surveys, LAL-Orsay, p. 243

Pratt, M. R., et al. 1996, in Proc. IAU Symp. 173, Astrophysical Applications of Gravitational Lensing, p. 221, Eds. C. S. Kochanek \& J. N. Hewitt, (Dordrecht: Kluwer Academic Publishers)

Racine, R. 1991, AJ, 102, 454

Rauch, K. P., \& Blandford, R. D. 1991, ApJ, 381, L39

Sasselov, D. 1996, in Proc. IAP Colloquium 12, Variable Stars and the Astrophysical Returns of Microlensing Surveys, eds. R. Ferlet and J. P. Maillard, (Paris: Editions Frontieres)

Sasselov, D., Heyrovský, D., \& Loeb, A. 1997, in preparation

Schild, R. E., \& Smith, R. C. 1991, AJ, 101, 813

Schneider, P., Ehlers, J., \& Falco, E. E. 1992, Gravitational Lenses (Berlin: Springer-Verlag)

Schneider, P., \& Wambsganss, J. 1990, A\&A, 237, 42

Steidel, C. C., Pettini, M., Dickinson, M., \& Persson, S. E. 1994, AJ 108, 2046 
Steidel, C. C., Bowen, D. V., Blades, J. C., \& Dickinson, M. 1995, ApJ, 440, L45

Steidel, C. C., Giavalisco, M., Dickinson, M., \& Adelberger, K. L. 1996, AJ, 112, 352

Tanaka, Y., et al. 1995, Nature, 375, 659

Udalski, A., et al. 1994, Acta Astron., 44, 165

Wambsganss, J. 1990, Gravitational Microlensing, report MPA 550, Garching

Wambsganss, J., \& Kundic, T. 1995, ApJ, 450, 19

Wambsganss, J., Paczyński, B., \& Schneider, P. 1990, ApJ, 358, L33

Warner, B. 1995, Cataclysmic Variable Stars, (Cambridge: Cambridge Univ. Press)

Witt, H. J. , \& Mao, S. 1994, ApJ, 430, 505 


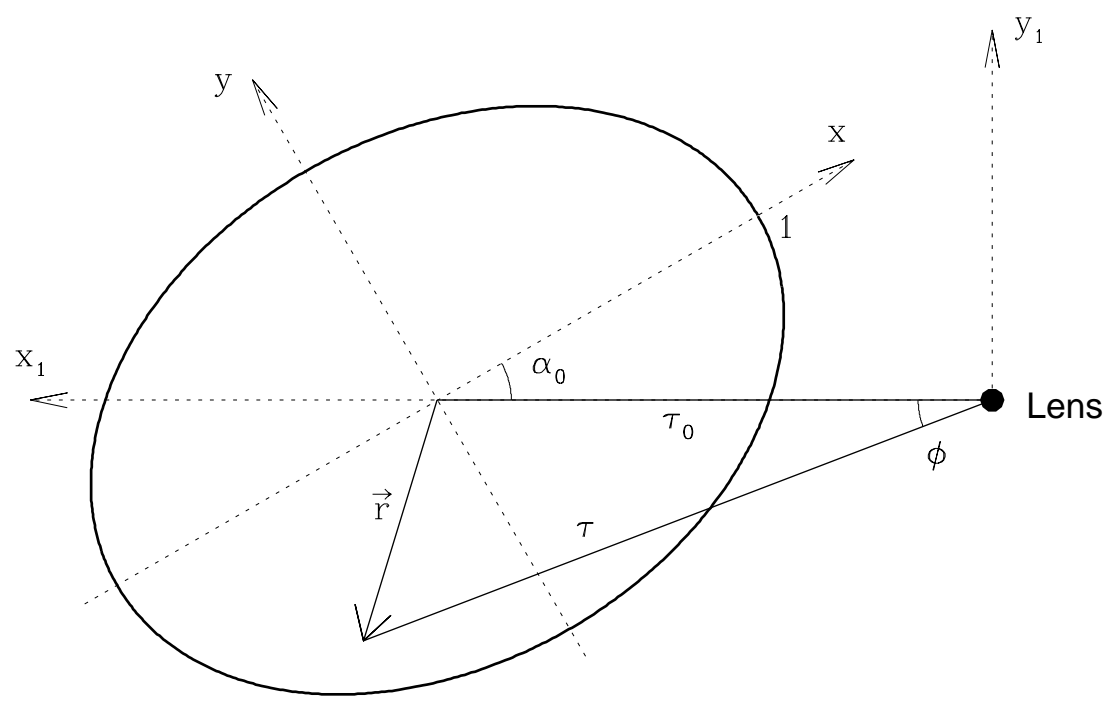

Fig. 1. - Geometry of the lens and an elliptical source projected on the sky. $\vec{r}$ marks an arbitrary point on the source. 


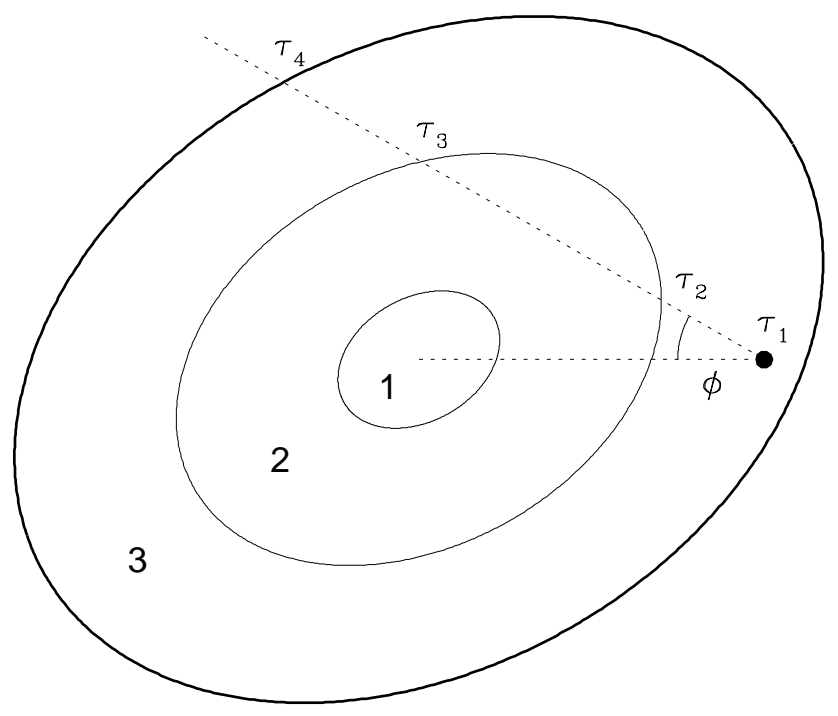

Fig. 2. - Sketch of the integration method geometry. The source is divided into three bands, each of which is characterized by its separate section of the brightness profile interpolation. For the angle $\phi$ in this figure, the band sequence is $\left\{s_{1}, s_{2}, s_{3}\right\}=\{3,2,3\}$ and $\tau_{1}=0, \tau_{4}=\tau_{+}(\phi)$. 


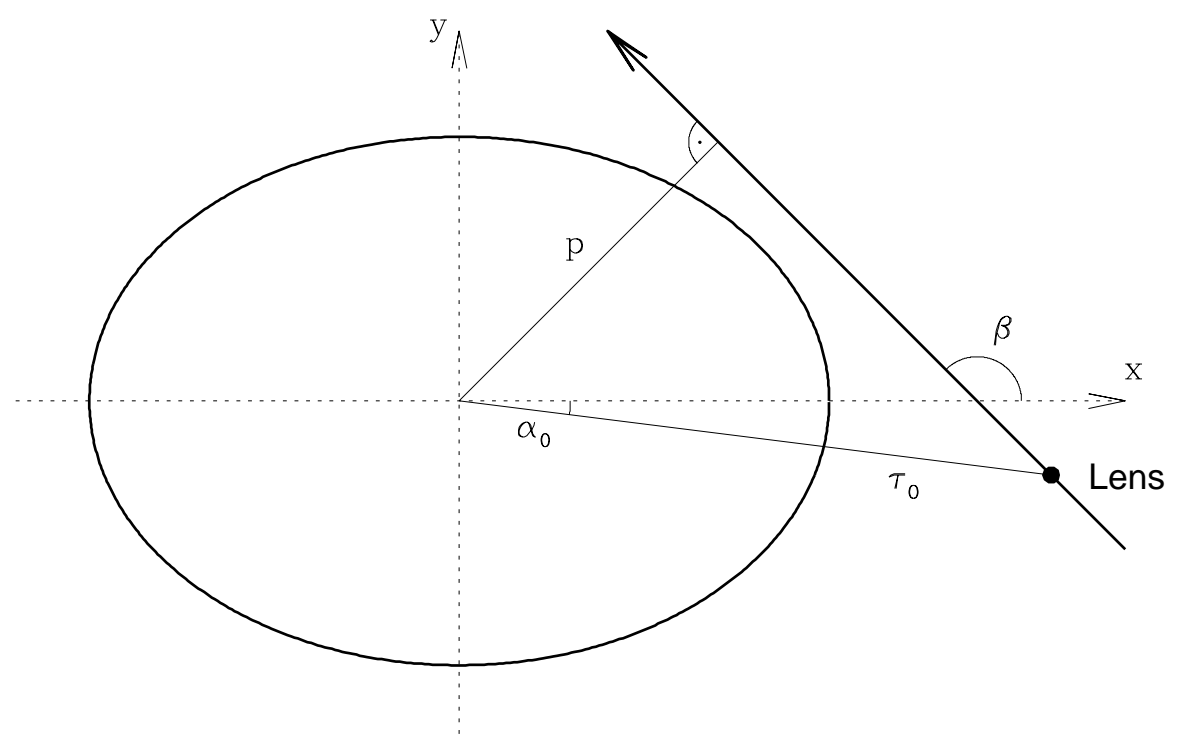

Fig. 3. - Trajectory of a lens in linear motion with respect to the source. 


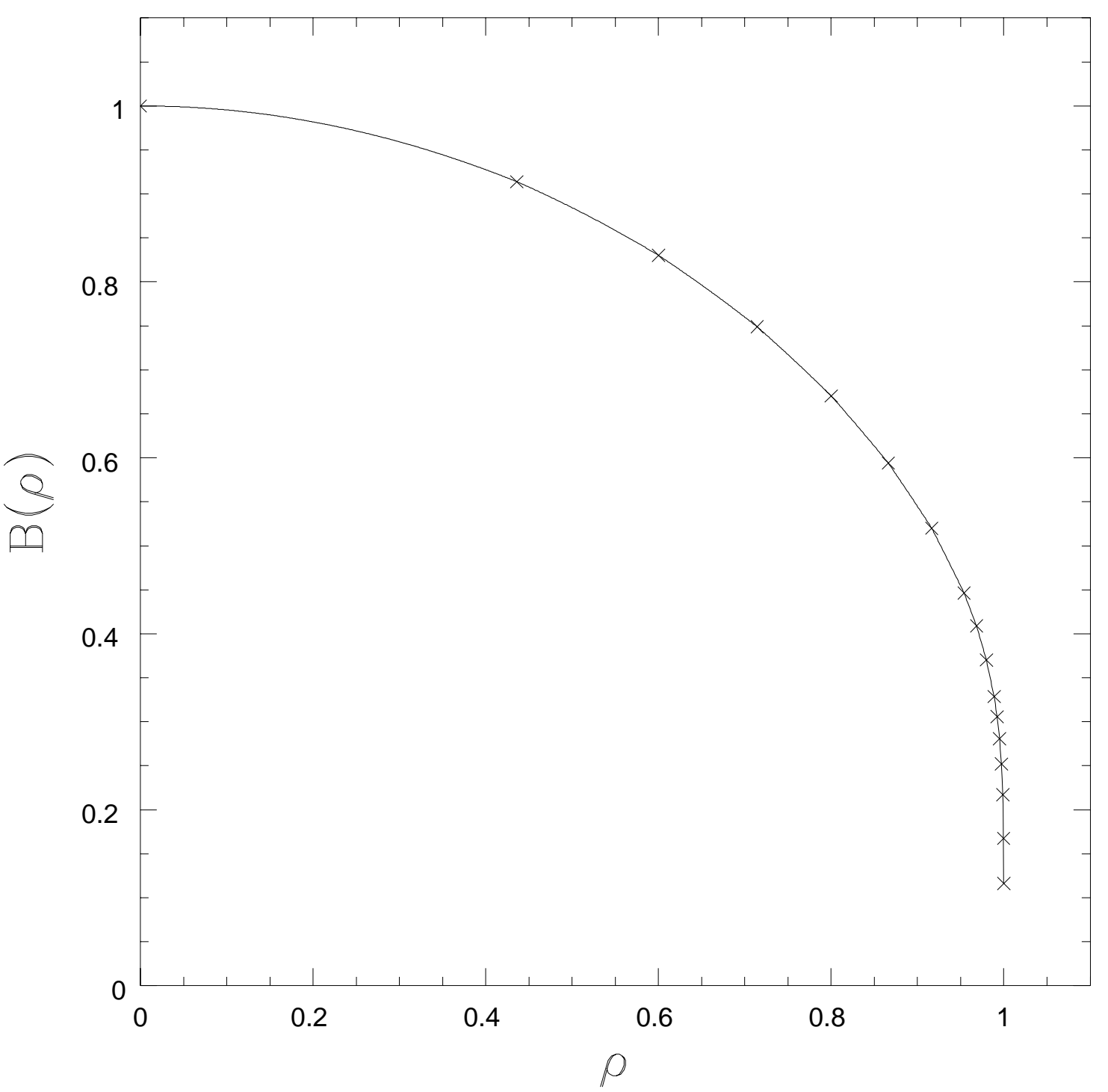

Fig. 4.- Brightness profile of the MACHO 95-30 source in the R-band, normalized by its central value. The profile is based on a model atmosphere calculated by Sasselov (1996). 


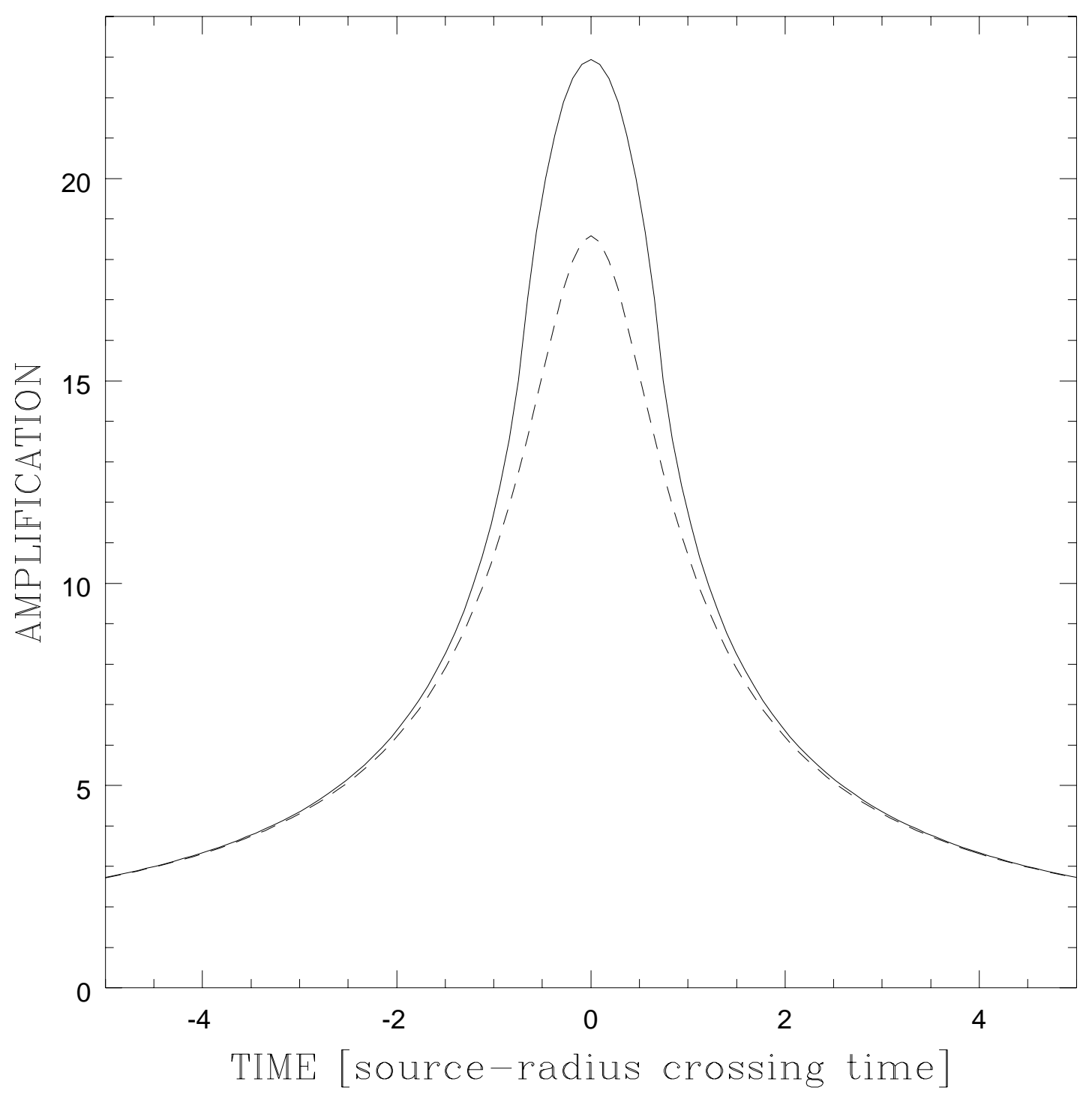

Fig. 5.- Simulated lightcurve of the MACHO 95-30 event (solid line), using the brightness profile in Figure 4. The lens was chosen to have an Einstein radius $\epsilon \approx 13$ and an impact parameter $p \approx 0.7$. For comparison, the dashed curve shows the point-source lightcurve for the same event parameters. 


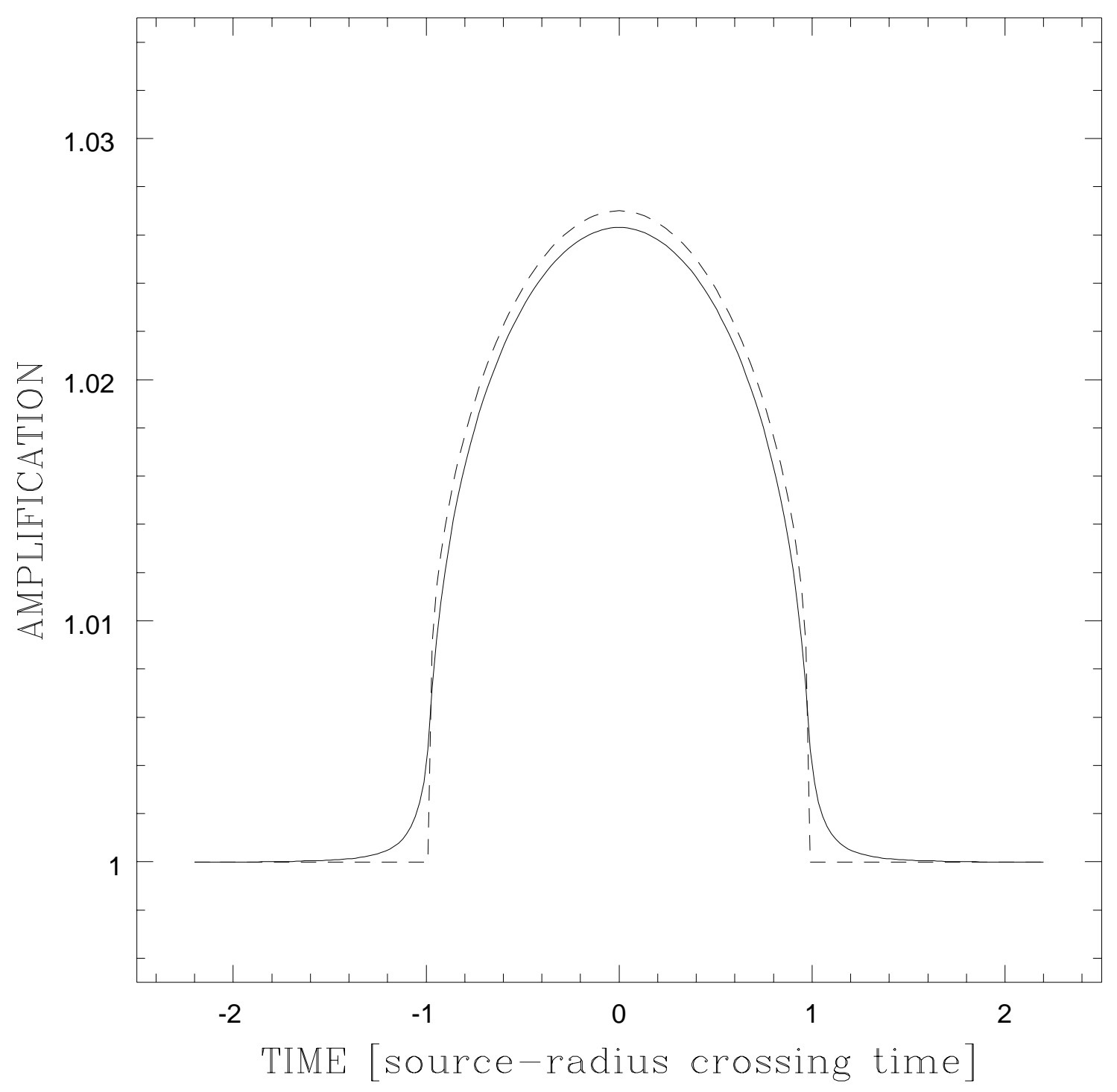

Fig. 6.- Lightcurve of the MACHO 95-30 source for a lens with an Einstein radius $\epsilon=0.1$ and an impact parameter $p=0.2$. The exact lightcurve (solid curve) is compared to its leading order approximation in the low lensing-mass limit (dashed curve). The brightness profile of the source was taken from Figure 4. 


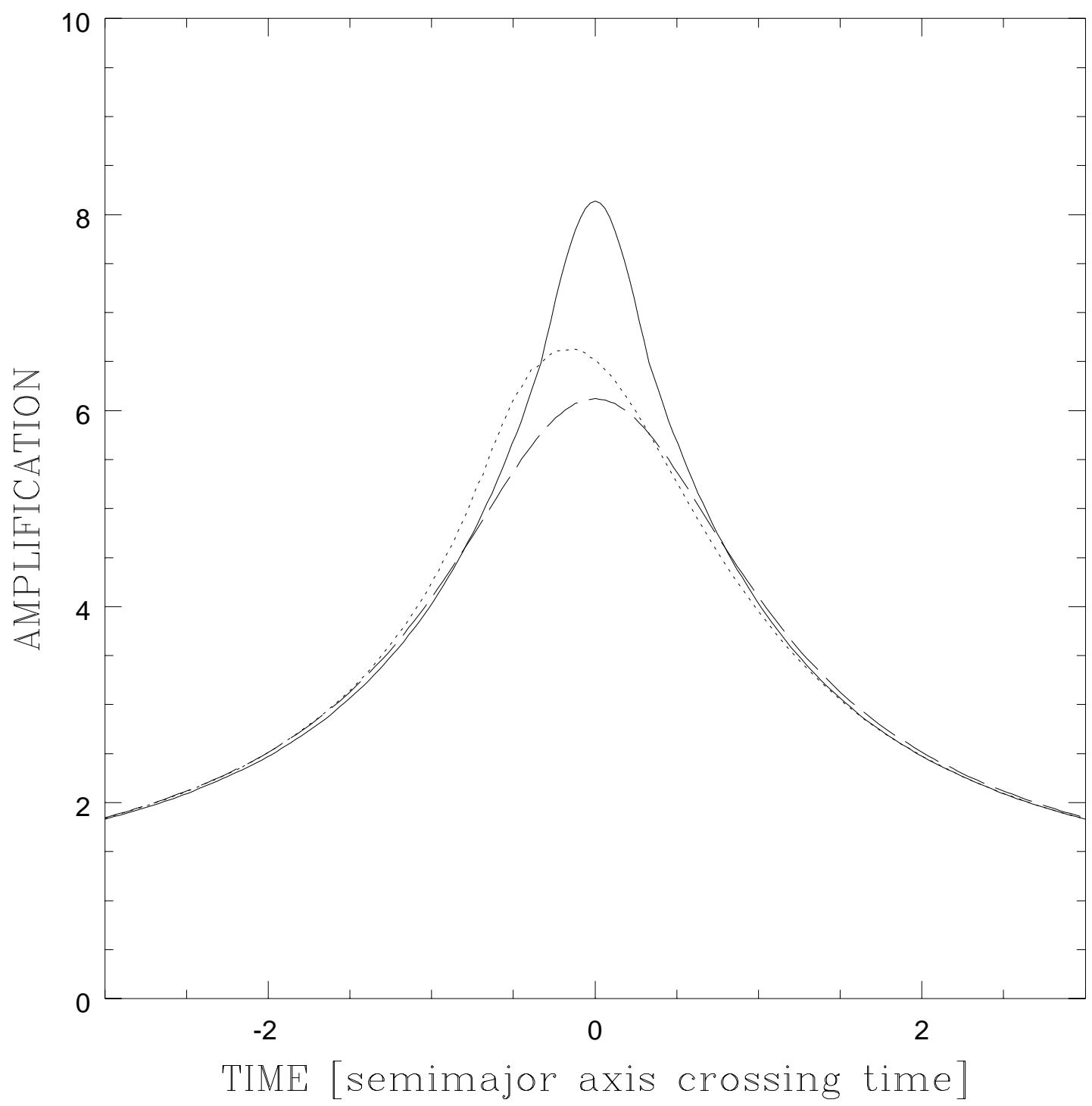

Fig. 7. - Lightcurves for an accretion disk inclined by an angle $i=60^{\circ}$, which is microlensed by a lens with an Einstein radius $\epsilon=5$. The three curves correspond to events with the same impact parameter $p=0.8$, but with a different lens orientation. The solid line corresponds to a trajectory parallel to the minor axis of the source $\left(\beta=90^{\circ}\right)$, the dotted line to a trajectory diagonal to the axes $\left(\beta=135^{\circ}\right)$, and the dashed line to a trajectory parallel to the major axis $\left(\beta=180^{\circ}\right)$. Zero time corresponds to closest approach in all cases. 


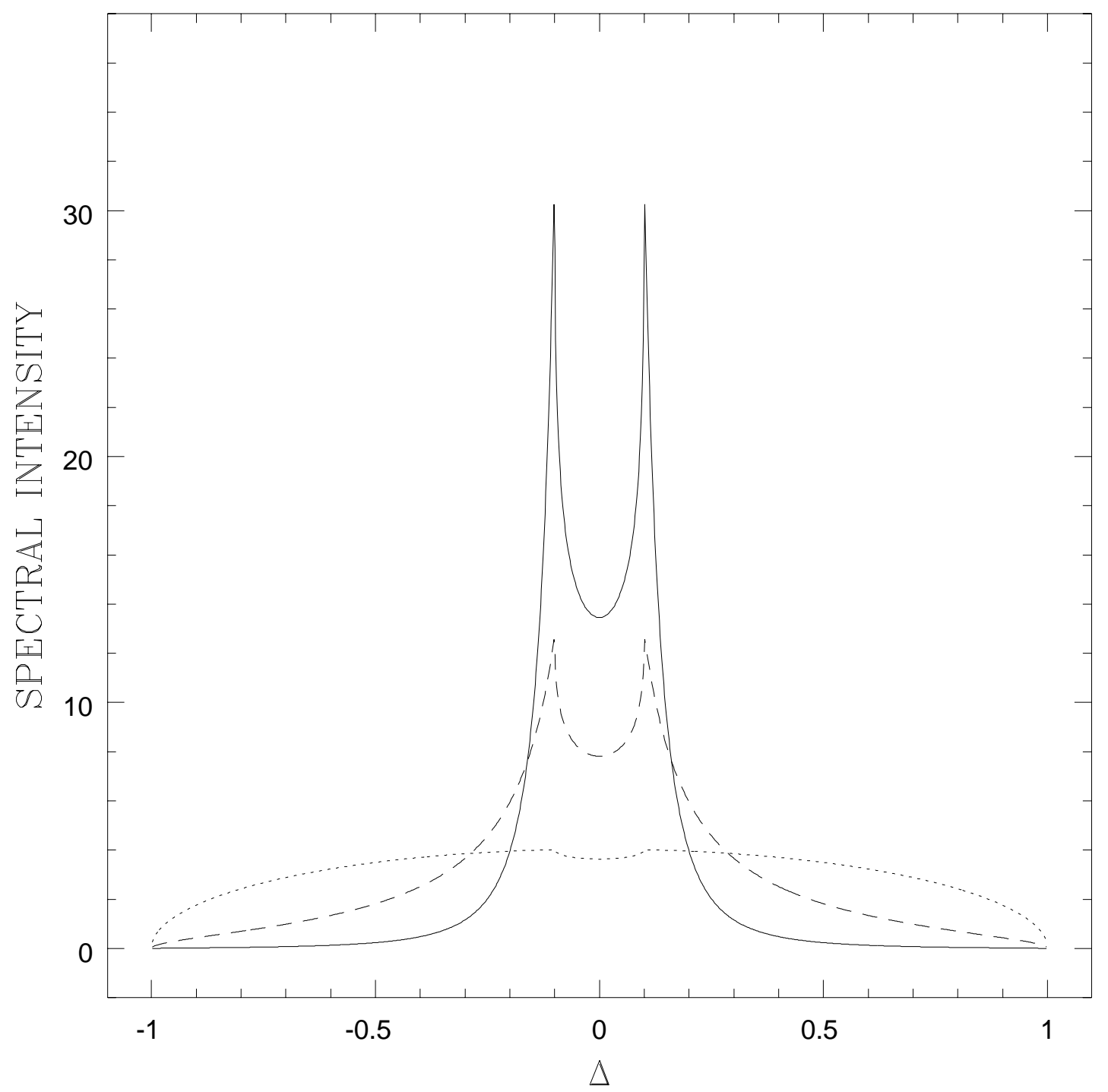

Fig. 8. - Dependence of the unlensed profile of a spectral line emitted by a Keplerian disk, on the radial power-law index of the disk emissivity. The different line profiles correspond to power-law indices of $n=-1$ (solid line), $n=-2$ (dashed line) and $n=-3$ (dotted line), for a disk with an inner radius $\rho_{i n}=0.01$ and inclination $i=60^{\circ}$. The vertical axis is in arbitrary units. 


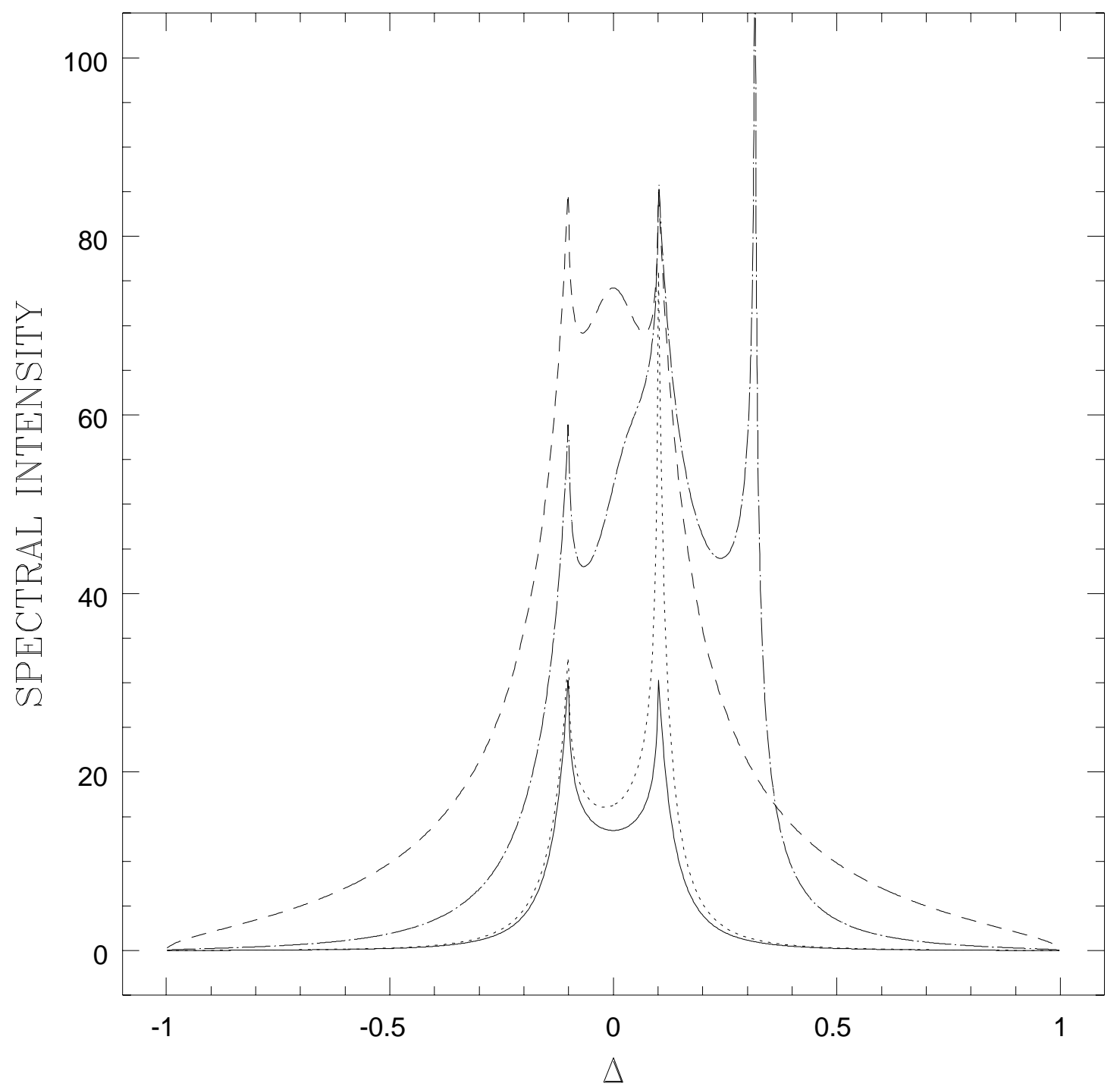

Fig. 9.- Effect of microlensing on the line profile for a disk with $n=-1$ in Figure 8, and for various lens positions along the major axis of the projected disk. The lens has an Einstein radius $\epsilon=1$, and is located at $\tau_{0}=0$ (dashed line), $\tau_{0}=0.1$ (dot-dashed), and $\tau_{0}=1.2$ (dotted). The unlensed profile is plotted for comparison (solid line). 


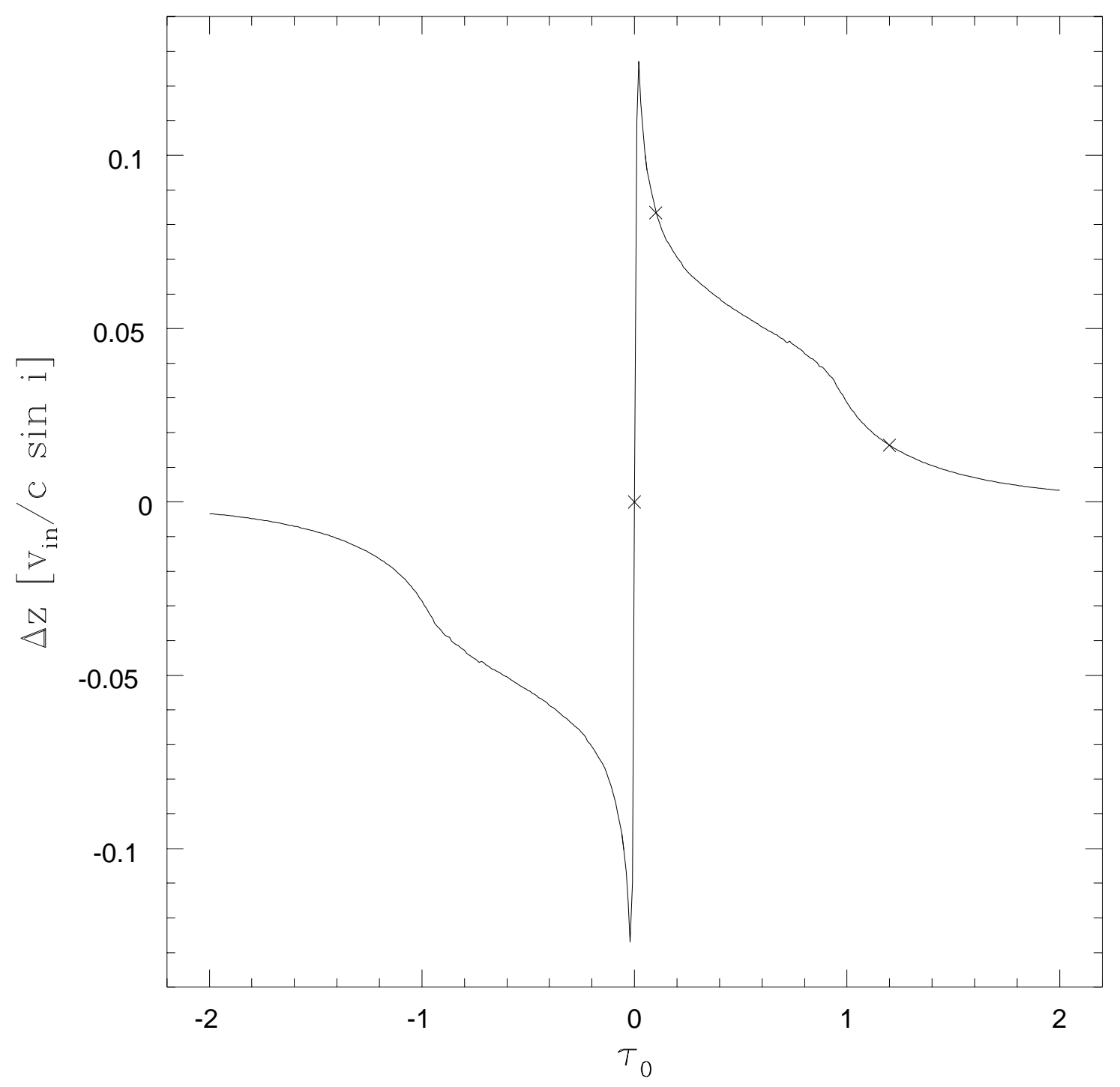

Fig. 10.- Change in mean redshift of the emitted line as a function of lens position $\tau_{0}$ along the major axis of the projected disk. The change in redshift is plotted in units of maximum Doppler shift from the inner edge of the unlensed disk. Disk parameters are the same as in Figures 8 and 9. The three marked points correspond to the lens positions for the line profiles in Figure 9. 